\begin{tabular}{|c|l|}
\hline Title & Quantum phase transition in a disordered long-range transverse Ising antiferromagnet \\
\hline Author(s) & Chandra, A njan Kumar; Inoue, Jun-ichi; Chakrabarti, Bikas K. \\
\hline Citation & $\begin{array}{l}\text { Physical review. E, Statistical, nonlinear, and soft matter physics, 81(2), 021101 } \\
\text { https://doi.org/L0.1103/PhysRevE.81.021101 }\end{array}$ \\
\hline Issue Date & 2010-02 \\
\hline Doc URL & http://hdl.handle.net/2115/42603 \\
\hline Rights & ○ 2010 A merican Physical Society \\
\hline Type & article \\
\hline File Information & e021101.pdf \\
\hline
\end{tabular}

Instructions for use 


\title{
Quantum phase transition in a disordered long-range transverse Ising antiferromagnet
}

\author{
Anjan Kumar Chandra, ${ }^{1, *}$ Jun-ichi Inoue, ${ }^{2, \dagger}$ and Bikas K. Chakrabarti, ${ }^{1, \dagger}$ \\ ${ }^{1}$ Centre for Applied Mathematics and Computational Science and Theoretical Condensed Matter Physics Division, \\ Saha Institute of Nuclear Physics, 1/AF Bidhannagar, Kolkata 700064, India \\ ${ }^{2}$ Complex Systems Engineering, Graduate School of Information Science and Technology, Hokkaido University, \\ N14-W9, Kita-ku, Sapporo 060-0814, Japan
}

(Received 9 December 2009; published 1 February 2010)

\begin{abstract}
We consider a long-range Ising antiferromagnet put in a transverse field (LRTIAF) with disorder. We have obtained the phase diagrams for both the classical and quantum cases. For the pure case applying quantum Monte Carlo method, we study the variation in order parameter (spin correlation in the Trotter direction), susceptibility, and average energy of the system for various values of the transverse field at different temperatures. The antiferromagnetic order is seen to get immediately broken as soon as the thermal or quantum fluctuations are added. We discuss generally the phase diagram for the same LRTIAF model with perturbative Sherrington-Kirkpatrick-type disorder. We find that while the antiferromagnetic order is immediately broken as one adds an infinitesimal transverse field or thermal fluctuation to the pure LRTIAF system, an infinitesimal SK spin-glass disorder is enough to induce a stable glass order in the LRTIAF. This glass order eventually gets destroyed as the thermal or quantum fluctuations are increased beyond their threshold values and the transition to paramagnetic phase occurs. Analytical studies for the phase transitions are discussed in details in each case. These transitions have been confirmed by applying classical and quantum Monte Carlo methods. We show here that the disordered LRTIAF has a surrogate incubation property of the SK spin glass phase.
\end{abstract}

DOI: 10.1103/PhysRevE.81.021101

PACS number(s): $64.70 . \mathrm{Tg}, 75.10 . J \mathrm{~m}, 75.10 . \mathrm{Nr}$

\section{INTRODUCTION}

Quantum phases in frustrated systems are being intensively investigated these days, in particular in the context of quantum spin-glass (SG) and quantum axial next-nearestneighbor Ising (ANNNI) models [1-6]. Here we study in general the long-range Ising antiferromagnet put under transverse field (LRTIAF) with disorder in cooperative interactions superposed on it. We study here extensively, both analytically as well as numerically, the phase diagram for this model. As a special case, we also studied the pure long-range transverse Ising antiferromagnet model (i.e., no disorder).

The finite-temperature properties of sublatticedecomposed version of the pure limit of this model were already considered earlier $[7,8]$. The quantum phase transition and entanglement properties of the full long-range model at zero temperature have also been studied [9].

Here we present some results obtained by applying analytical as well as Monte Carlo techniques [10] to the general full long-range model at finite temperatures and transverse fields. We observe indications of a very unstable quantum antiferromagnetic (AF) phase (50\% spin up, 50\% spin down, without any sublattice structure) in the pure LRTIAF model, where the antiferromagnetically ordered phase gets destabilized by both infinitesimal thermal (classical) as well as quantum fluctuations (due to tunneling or transverse field) and the system becomes disordered or goes over to the paramagnetic phase [11]. This kind of phase transition has also been studied by perturbative treatment [12].

\footnotetext{
*anjan.chandra@saha.ac.in

†_inoue@ complex.eng.hokudai.ac.jp

tbikask.chakrabarti@saha.ac.in
}

When a little spin-glass-like disorder is incorporated with this pure LRTIAF model, the frustration is seen to destabilize the AF phase and stabilize a spin-glass order. To check how this "liquid"-like antiferromagnetic phase of the pure LRTIAF gets "frozen" into spin-glass phase when a little disorder is added, we study in general the LRTIAF Hamiltonian with a coupling with the Sherrington-Kirkpatrick (SK) spinglass Hamiltonian and study this entire system's phasetransition behavior induced by both thermal and tunneling fields. Indeed, stable SK-like spin-glass phase is observed for both thermal and quantum fluctuations below finite threshold values [11].

This paper is organized in the following manner. In Sec. II, we present the quantum LRTIAF model with SK disorder. Then in Secs. II A-II D, we discuss the analytical studies and simulations for some special cases of this general model. In Sec. II A, we consider the quantum LRTIAF model without disorder, in Sec. II B, the classical model (i.e., without the transverse field), in Sec. II C, the quantum model at finite temperature, and in Sec. II D, the quantum model at zero temperature. The detail calculation of free energy is given in Appendix A and an exact analysis at $T=0$ is given in Appendix B In Sec. III, we present some discussions on our results.

\section{LRTIAF WITH SK DISORDER}

The general model we study here is given by the following Hamiltonian:

$$
H=-\frac{1}{N} \sum_{i j(j>i)}\left(J_{0}+\widetilde{J} \tau_{i j}\right) \sigma_{i}^{z} \sigma_{j}^{z}-h \sum_{i=1}^{N} \sigma_{i}^{z}-\Gamma \sum_{i} \sigma_{i}^{x},
$$

where $J_{0}$ is the parameter controlling the strength of the antiferromagnetic bias and $\widetilde{J}$ is an amplitude of the disorder $\tau_{i j}$ 
in each pair interaction. $h$ and $\Gamma$ denote, respectively, the longitudinal and transverse fields. The $\Gamma$ controls the quantum-mechanical fluctuation. Here, $\sigma^{x}$ and $\sigma^{z}$ denote the $x$ and $z$ components of the $N$ Pauli spins

$$
\sigma_{i}^{z}=\left(\begin{array}{cc}
1 & 0 \\
0 & -1
\end{array}\right), \quad \sigma_{i}^{x}=\left(\begin{array}{ll}
0 & 1 \\
1 & 0
\end{array}\right), \quad i=1,2, \ldots, N .
$$

As such, the model has a fully frustrated (infinite-range or infinite-dimensional) cooperative term. When we assume that the disorder $\tau_{i j}$ obeys a Gaussian with mean zero and variance unity, the new variable $J_{i j} \equiv J_{0}+\widetilde{J} \tau_{i j}$ follows the Gaussian distribution, $P\left(J_{i j}\right)=\exp \left[-\left(J_{i j}-J_{0}\right)^{2} / 2 \widetilde{J}^{2}\right] / \sqrt{2 \pi} \widetilde{J}$. Therefore, we obtain the "pure" antiferromagnetic Ising model with infinite-range interactions when we consider the limit $\widetilde{J} \rightarrow 0$, keeping $J_{0}<0$. Of course, the model with $J_{0}$ $>0$ and $\Gamma=0$ is identical to the classical SK model and with $J_{0}<0$ and $\Gamma=0$, it is the LRIAF model.

For an analytic (mean-field) study of the model, we define an effective magnetic field $\vec{h}_{e f f}$ at each site, which is a resultant of the average cooperation enforcement in the $z$ direction and the applied transverse field in the $x$ direction, so that the above Hamiltonian can be written as

$$
H=\vec{h}_{e f f} \cdot \sum_{i=1}^{N} \vec{\sigma}_{i},
$$

where

$$
\vec{\sigma}_{i}=\sigma_{i}^{z} \hat{z}+\sigma_{i}^{x} \hat{x}
$$

and

$$
\begin{gathered}
\vec{h}_{e f f}=\left(\vec{h}_{e f f}\right)^{z} \hat{z}+\left(\vec{h}_{e f f}\right)^{x} \hat{x}=\left(h+J_{0} m^{z}+\widetilde{J} \sqrt{q y}\right) \hat{z}+\Gamma \hat{x}, \\
\left|\vec{h}_{e f f}\right|=\sqrt{\left(h+J_{0} m^{z}+\widetilde{J} \sqrt{q} y\right)^{2}+\Gamma^{2}} .
\end{gathered}
$$

This replacement of $\sigma_{j}^{z}$ by its average value $\left\langle\sigma_{j}^{z}\right\rangle \equiv m^{z}$ in $\left(\vec{h}_{e f f}\right)^{z}$ should be valid for this infinite-range model (see Appendix $\mathrm{A}$ for its much more precise description under replica-symmetric theory). The Gaussian distributed random field $\sqrt{q} y$ comes from the local-field fluctuation (see, e.g., [13]) given by the spin-glass order parameter (see Appendix A for details). The average magnetization is then given by

$$
\vec{m}=\frac{\operatorname{Tr} \vec{\sigma} e^{-\beta H}}{\operatorname{Tr} e^{-\beta H}}=\left(\tanh \beta\left|\vec{h}_{e f f}\right|\right) \frac{\vec{h}_{e f f}}{\left|\vec{h}_{e f f}\right|}
$$

and hence we have

$$
\begin{aligned}
& m^{z}=\int_{-\infty}^{\infty} D y \frac{J_{e f f}}{\sqrt{J_{e f f}^{2}+\Gamma^{2}}} \tanh \beta \sqrt{J_{e f f}^{2}+\Gamma^{2}}, \\
& m^{x}=\int_{-\infty}^{\infty} D y \frac{\Gamma}{\sqrt{J_{e f f}^{2}+\Gamma^{2}}} \tanh \beta \sqrt{J_{e f f}^{2}+\Gamma^{2}},
\end{aligned}
$$

$$
q=\int_{-\infty}^{\infty} D y\left\{\frac{J_{e f f}}{\sqrt{J_{e f f}^{2}+\Gamma^{2}}}\right\}^{2} \tanh ^{2} \beta \sqrt{J_{e f f}^{2}+\Gamma^{2}},
$$

where $J_{e f f}=h+J_{0} m^{z}+\widetilde{J} \sqrt{q} y, m \equiv N^{-1} \sum_{i}\left\langle\sigma_{i}^{z}\right\rangle$ is the magnetization, and $q \equiv N^{-1} \Sigma_{i}\left\langle\sigma_{i}^{z}\right\rangle^{2}$ is the spin-glass order parameter. We defined $D y \equiv d y \exp \left(-y^{2} / 2\right) / \sqrt{2 \pi}$. In Appendix A, we confirm that the above mean-field equations are identical to the results obtained by the replica-symmetric theory at the ground state $(\beta=\infty)$.

For the antiferromagnetic $\left(J_{0}<0\right)$ and/or the spin-glass phase (with $h=0$ ), $m^{z}=0$ is the only solution. We then have

$$
\begin{aligned}
& m^{x}=\int_{-\infty}^{\infty} D y \frac{\Gamma}{\sqrt{(\tilde{J} \sqrt{q} y)^{2}+\Gamma^{2}}} \tanh \beta \sqrt{(\tilde{J} \sqrt{q} y)^{2}+\Gamma^{2}}, \\
& q=\int_{-\infty}^{\infty} D y\left\{\frac{\widetilde{J} \sqrt{q} y}{\sqrt{(\widetilde{J} \sqrt{q} y)^{2}+\Gamma^{2}}}\right\}^{2} \tanh ^{2} \beta \sqrt{(\widetilde{J} \sqrt{q} y)^{2}+\Gamma^{2}} .
\end{aligned}
$$

\section{A. Pure LRTIAF model}

The Hamiltonian of the infinite-range quantum Ising antiferromagnet (without any spin-glass disorder) is

$$
H \equiv H^{(C)}+H^{(T)}=-\frac{J_{0}}{N} \sum_{i, j(>i)=1}^{N} \sigma_{i}^{z} \sigma_{j}^{z}-h \sum_{i=1}^{N} \sigma_{i}^{z}-\Gamma \sum_{i=1}^{N} \sigma_{i}^{x},
$$

where $J_{0}$ denotes the long-range antiferromagnetic $\left(J_{0}<0\right)$ exchange constant. We have denoted the cooperative term of $H$ (including the external longitudinal field term) by $H^{(C)}$ and the transverse field part as $H^{(T)}$. As such, the model has a fully frustrated (infinite-range or infinite-dimensional) cooperative term.

\section{Analytical studies}

For $h=0$, the Hamiltonian can be written as

$$
H=-\frac{J_{0}}{N} \sum_{i, j(>i)=1}^{N} \sigma_{i}^{z} \sigma_{j}^{z}-\Gamma \sum_{i=1}^{N} \sigma_{i}^{x}=-\frac{J_{0}}{N}\left(\sum_{i=1}^{N} \sigma_{i}^{z}\right)^{2}-\Gamma \sum_{i=1}^{N} \sigma_{i}^{x} .
$$

Without the disorder term, the $\vec{h}_{\text {eff }}$ in Eq. (3a) gets modified to

$$
\vec{h}_{e f f}=J_{0} m^{z} \hat{z}+\Gamma \hat{x} .
$$

With this modified field, the expressions of $m^{z}$ and $m^{x}$ become

$$
\begin{aligned}
& m^{z}=\left[\tanh \beta \sqrt{\left(J_{0} m^{z}\right)^{2}+\Gamma^{2}}\right] \frac{J_{0} m^{z}}{\sqrt{\left(J_{0} m^{z}\right)^{2}+\Gamma^{2}}}, \\
& m^{x}=\left[\tanh \beta \sqrt{\left(J_{0} m^{z}\right)^{2}+\Gamma^{2}}\right] \frac{\Gamma}{\sqrt{\left(J_{0} m^{z}\right)^{2}+\Gamma^{2}}} .
\end{aligned}
$$

When $J_{0}<0$, then $m^{z}=0$ is the only solution of Eq. (13a). At zero temperature and at zero longitudinal and transverse 
fields, the $H^{(C)}$ would prefer the spins to orient in $\pm z$ directions only with zero net magnetization in the $z$ direction. This antiferromagnetically ordered state is completely frustrated and highly degenerate. Switching on the transverse field $\Gamma$ would immediately induce all the spins to orient in the $x$ direction (losing the degeneracy) corresponding to a maximum of the kinetic-energy term and this discontinuous transition to the paramagnetic phase occurs at $\Gamma=0$ (see Appendix B for an exact result at $T=0$ ). However, at any finite temperature, the entropy term coming from the extreme degeneracy of the antiferromagnetically ordered state and the close-by excited states does not seem to induce a stability of this phase.

\section{Monte Carlo simulation}

This Hamiltonian (10) can be mapped to a $(\infty+1)$-dimensional classical Hamiltonian $[10,14]$ using the Suzuki-Trotter formula. The effective Hamiltonian can be written as (for $J_{0}=-1$ )

$$
\begin{aligned}
\mathcal{H}= & -\frac{1}{N M} \sum_{i, j(>i)=1}^{N} \sum_{k=1}^{M} \sigma_{i, k} \sigma_{j, k}-\frac{h}{M} \sum_{i=1}^{N} \sum_{k=1}^{M} \sigma_{i, k} \\
& +B \sum_{i=1}^{N} \sum_{k=1}^{M} \sigma_{i, k} \sigma_{i, k+1},
\end{aligned}
$$

where

$$
B=(1 / 2) \ln [\operatorname{coth}(\Gamma / M T)] .
$$

Here, $M$ is the number of Trotter replicas and $k$ denotes the $k$ th row in the Trotter direction. $B$ denotes the nearestneighbor interaction strength along the Trotter direction. We have studied the system for $N=100$. Because of the diverging growth of interaction $B$ for very low values of $\Gamma$ and also for high values of $M$ and the consequent nonergodicity (the system relaxes to different states for identical thermal and quantum parameters due to frustrations, starting from different initial configurations), we have kept the value of $M$ at a fixed value of 5. This choice of $M$ value helped satisfying the ergodicity of the system up to very low values of the transverse field at the different temperatures considered, $T=0.10$, 0.20 , and 0.30 . Starting from a random initial configuration (including all up or 50-50 up-down configurations), we follow the time variations of different quantities until they relax and study the various quantities after they relax.

We studied results for three different temperatures, $T$ $=0.10,0.20$, and 0.30, and all the results are for $N=100$ and 200 and $M=5$. We estimated the following quantities after relaxation:

(i) Correlation along Trotter direction $(\widetilde{q})$. We studied the variation in the order parameter

$$
\tilde{q}=\frac{1}{N M} \sum_{i=1}^{N} \sum_{k=1}^{M}\left\langle\sigma_{i, k} \sigma_{i, k+1}\right\rangle,
$$

which is the first neighbor correlation along Trotter direction. Here, $\langle\cdots\rangle$ indicates the average over initial spin configurations. This quantity $\tilde{q}$ shows a smooth vanishing behavior. We consider this correlation $\tilde{q}$ as the order parameter for the

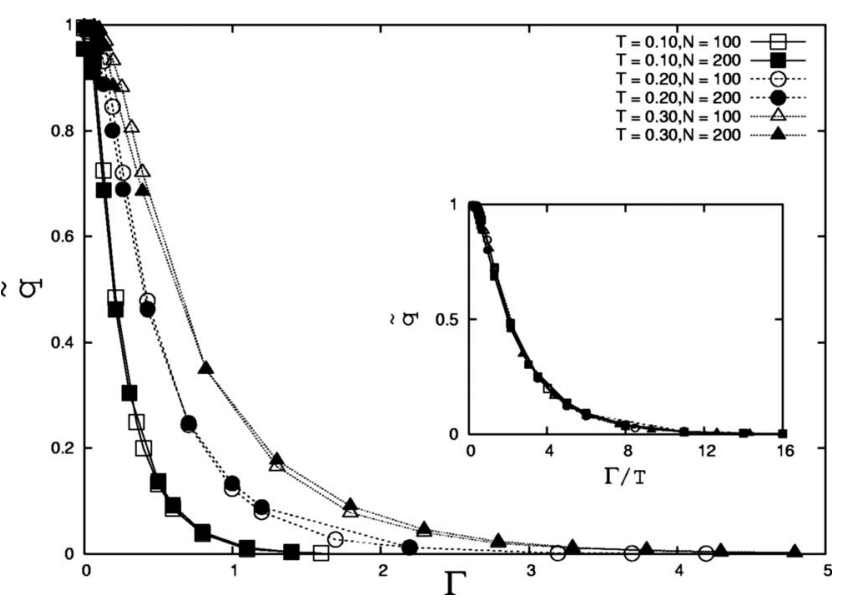

FIG. 1. Variation in the order parameter $\widetilde{q}$ (correlation in the Trotter direction) with transverse field $\Gamma$ for $T=0.10,0.20$, and 0.30 $(h=0)$ for two different system sizes $(N=100$ and 200$) . \tilde{q}=0$ for large $\Gamma$. Inset shows the plot of $\tilde{q}$ against the scaled variable $\Gamma / T$.

transition at $\Gamma_{c}$. A larger transverse field is needed for the vanishing of the order parameter for larger temperature. The observed values (see Fig. 1) of $\Gamma_{c}$ are $\simeq 1.6,2.2$, and 3.0 for $T=0.1,0.2$, and 0.3 , respectively. As shown in the inset, a unique data collapse occurs when $\widetilde{q}$ is plotted against $\Gamma / T$ and one seems to get the complete disorder immediately as the scaling does not involve any finite value $T_{c}$. This is consistent with the observations in the next section.

(ii) Susceptibility $(\chi)$. The longitudinal susceptibility $\chi$ $=(1 / N M) \partial\left[\sum_{i, k}\left\langle\sigma_{i, k}\right\rangle\right] / \partial h$, where $h(\rightarrow 0)$ is the applied longitudinal field, has also been measured. We went up to $h=0.1$ and estimated the $\chi$ values. As we increase the value of the transverse field $\Gamma$ from a suitably chosen low value, $\chi$ initially starts with a value almost equal to unity and then gradually saturates at lower values [corresponding to the classical system where $B=0$ in Eq. (14)] as $\Gamma$ is increased. Also at $\Gamma=0$, the classical values are indicated in Fig. 2. This saturation value of $\chi$ decreases with temperature. Again, the field at which the susceptibility saturates is the same as for the vanishing of the order parameter for each temperature.

(iii) Average energy $(E)$. We have measured the value of the cooperative energy for each Trotter index and then take its average $E$, i.e., $E=\left\langle H^{(C)}\right\rangle$ of Eq. (10) with $J_{0}=-1$. It initially begins with -1.0 and after a sharp rise, the average energy saturates, at large values of $\Gamma$, to values corresponding to the classical equilibrium energy $\left[E_{c l}\right.$ for $B=0$ in Eq. (14)] at those temperatures. Again, it takes larger values of $\Gamma$ at higher temperatures to achieve the classical equilibrium energy. At $\Gamma=0$, the corresponding classical values of $E$ are plotted in Fig. 3. The variations of all these quantities indicate that the "quantum order" disappears and the quantities reduce to their classical values (corresponding to $B=0$ ) for large values of the transverse field $\Gamma$.

The continuous-transition-like behavior seen from Fig. 1 can be justified from a mean-field analysis (see Appendix B). At finite temperature, it is the free energy that we have to minimize and the entropy term plays a crucial role. Minimization of free energy leads to an analytic variation in the total magnetization and no phase transition at any finite temperature. 


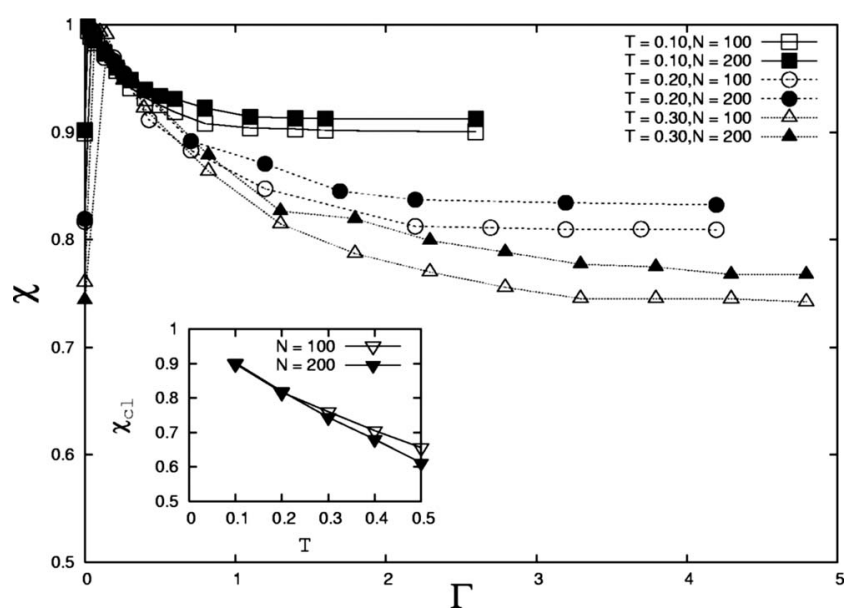

FIG. 2. Variation in the susceptibility $\chi$ with transverse field $\Gamma$ for $T=0.10,0.20$, and $0.30(h \leq 0.1)$ for two different system sizes $\left(N=100\right.$ and 200). The corresponding susceptibilities $\chi_{c l}$ for various temperatures for $N=100$ and 200 for the classical system are shown in the inset. $\chi$ converges to the classical values $\chi_{c l}$ for large $\Gamma$.

\section{B. LRIAF with disorder: Classical model at finite temperature}

\section{Analytical studies}

For the classical case, i.e., $\Gamma=0$, Eqs. (5) and (7) reduce to (for $h=0$ )

$$
\begin{aligned}
& m=\int_{-\infty}^{\infty} D y \tanh \beta\left(\tilde{J} \sqrt{q} y+J_{0} m\right), \\
& q=\int_{-\infty}^{\infty} D y \tanh ^{2}\left(\beta \tilde{J} \sqrt{q} y+J_{0} m\right),
\end{aligned}
$$

where $m^{z}$ has been replaced by $m$. For $J_{0}<0$, again we find that $m=0$ is only physical solution for all temperature regimes. This means that there are three possible phases:

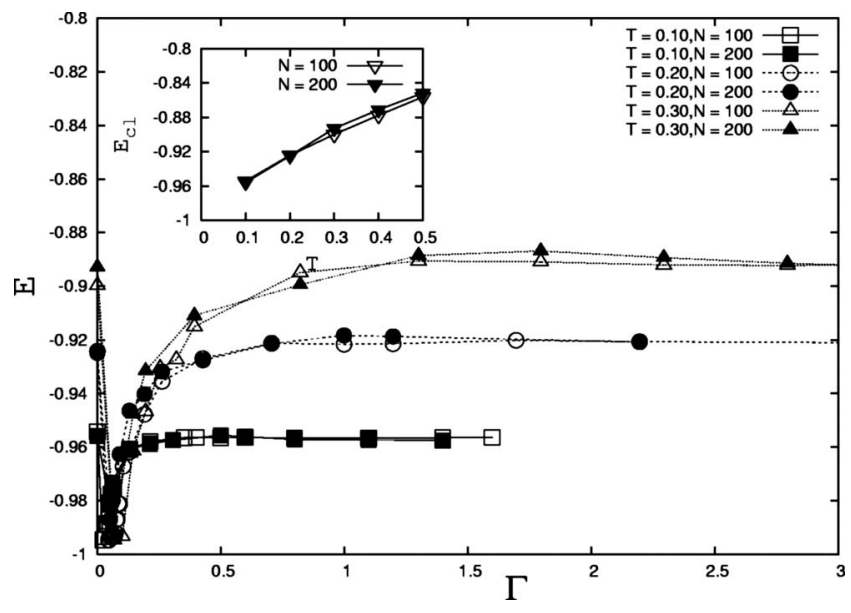

FIG. 3. Variation in average energy $E$ with transverse field $\Gamma$ for $T=0.10,0.20$, and $0.30(h=0)$ for two different values of $N($ $=100,200)$. The corresponding average energies $E_{c l}$ for various temperatures for $N=100$ and 200 are shown in the inset. $E$ converges to the classical values $E_{c l}$ for large $\Gamma$.

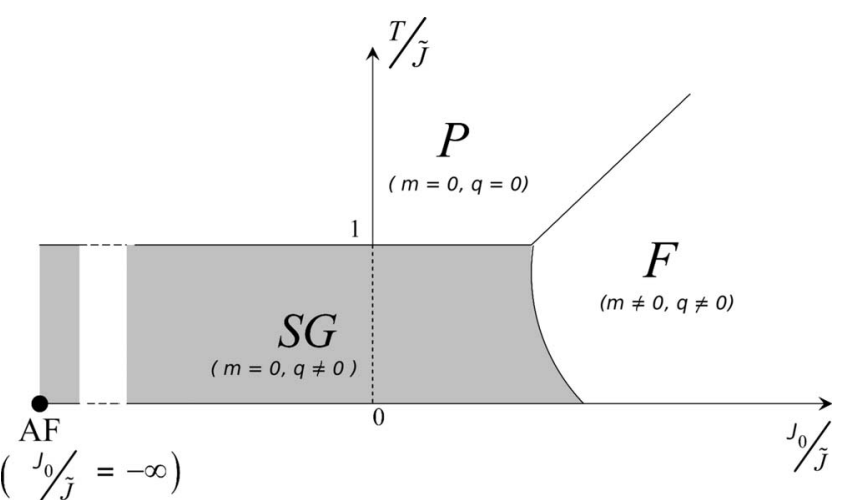

FIG. 4. Phase diagram of classical SK model [13] extended for antiferromagnetic bias. For $J_{0}<0$, there exists spin-glass phase below $T / \widetilde{J}=1$ and the critical temperature is independent of the strength of the antiferromagnetic bias $J_{0}$. For pure LRIAF at finite temperature $(T>0)$, the antiferromagnetic order disappears and the system changes to the paramagnetic phase. When we add an infinitesimal disorder $\widetilde{J}>0$, the antiferromagnetic order is broken down and the system suddenly gets frozen into a SG phase.

namely, the antiferromagnetic phase, the paramagnetic phase, and the spin-glass phase. In all these three phases, the magnetization $m$ is zero. To determine the critical point $T_{S G}$ at which the spin-glass transition takes place, we expand the equation with respect to $q$ for $q \simeq 0$ and $m=0$. In the limit of $\tilde{J} \ll 1$, we have

$$
q=\frac{(\beta \widetilde{J})^{2}-1}{2(\beta \widetilde{J})^{4}} .
$$

We therefore have $T_{S G}=\widetilde{J}$ and the critical point is independent of the antiferromagnetic bias $J_{0}$. This result means that the antiferromagnetic order can appear if and only if we set $J_{0}<0$ and $T / \widetilde{J}=0, J_{0} / \widetilde{J}=-\infty$. On the other hand, for $-\infty$ $<J_{0}<0$ at low-temperature regime $T<T_{S G}$, the spin-glass phase appears. We plot the phase diagram in Fig. 4. We also conclude that the system described by Hamiltonian (10) with $\Gamma=0$ is immediately frozen when we add any infinitesimal disorder $\widetilde{J}>0$.

From the viewpoint of the degeneracy of the spin configurations, we easily estimate the number of solution for the antiferromagnetic phase as $N ! /[(N / 2) !(N / 2) !] \simeq e^{0.693 N}[$ in the antiferromagnetic ground state, only $N / 2$ spins out of $N$ will have free choice (to be up or down) and the rest have to follow], which is larger than the number of the SK model $e^{0.199 N}[13]$. However, for the infinite-range antiferromagnetic model, the energy barrier between arbitrary configurations which gives the same lowest-energy states is of order 1 and there is no ergodicity breaking.

\section{Monte Carlo studies}

In order to study the effect of introducing disorder in the classical LRIAF model, we performed a Monte Carlo study with a system of $N=100$ spins. But the distribution function $(\widetilde{P})$ of disorder introduced here is different. Instead of a 
Gaussian distribution, we applied a binary distribution $( \pm J)$ with a probability $p$,

$$
\tilde{P}\left(J_{i j}\right)=p \delta\left(J_{i j}-J\right)+(1-p) \delta\left(J_{i j}+J\right) .
$$

In our study, we kept $J=1$ and $J_{0}=\bar{J}$, where $\bar{J}$ is the average interaction strength. Each of the Ising spins interacts with every other ferromagnetically with probability $p$ and antiferromagnetically with probability $1-p$. Therefore, $\bar{J}=2 p-1$ and $\widetilde{J}=2 \sqrt{p(1-p)}$. At the two limiting values of $p=0$ and $p$ $=1$, the system becomes purely antiferromagnetic (LRIAF) and purely ferromagnetic, respectively. Thus at these two limiting values of $p$, the system has no fluctuation at all $(\tilde{J}$ $=0)$. Whereas for $p=0.5$, the fluctuation is maximum $(\tilde{J}=1)$. So as predicted above, we need maximum thermal fluctuation to destroy the glassy phase.

To identify the glass phase, we considered a replica of the original system to be studied and evolved the two systems simultaneously by Monte Carlo technique. We also repeated the study for $N=50$ and 200 also. For all sizes, we obtained almost same phase diagram. We measured the average absolute value of the spin-spin correlations of the two systems (the original and the replica one) at different times for a certain temperature. Let us denote this parameter by $q$ and $\alpha$ and $\beta$ denotes the original system and the replica

$$
q=\left\langle\left|\frac{1}{N} \sum_{i=1}^{N} s_{i}^{\alpha} s_{i}^{\beta}\right|\right\rangle .
$$

We measure the average steady-state values of this parameter for various temperatures. The average is over different configurations. When $p$ is very high, i.e., when the number of ferromagnetic bonds is dominant, the ferromagnetic to paramagnetic transition can be identified easily by measuring the change in magnetization and divergence of susceptibility at the critical temperature. But for $p<0.5$ and slightly above 0.5 , the magnetization remains low for all temperatures and hence it is difficult to identify the existence of any glass phase or paramagnetic phase separately. The glass and paramagnetic phases can be distinguished by studying the steadystate values and fluctuation of $q$. We have studied for three different values of $p=0.20,0.30$, and 0.40 . For a particular value of $p$, up to a certain temperature, the value of $q$ remains high, indicating the spin-glass phase. The fluctuations in $q$ remain small. This implies that the flipping of spins is very low (freezing of spins). As the temperature is raised, the steady-state value of $q$ decreases gradually (inset of Fig. 5) but the fluctuation increases. At a certain temperature (glassparamagnetic phase-transition point), this fluctuation reaches maximum and above this temperature, the value of $q$ gradually goes to zero. With further increase of temperature, the fluctuation decreases, indicating a second-order glassparamagnetic phase transition (Fig. 5).

It can be observed from (Fig. 5) that as we increase the value of $p$ from 0 to 0.5 , the transition temperature increases. It is expected from our previous explanation, as $p$ approaches

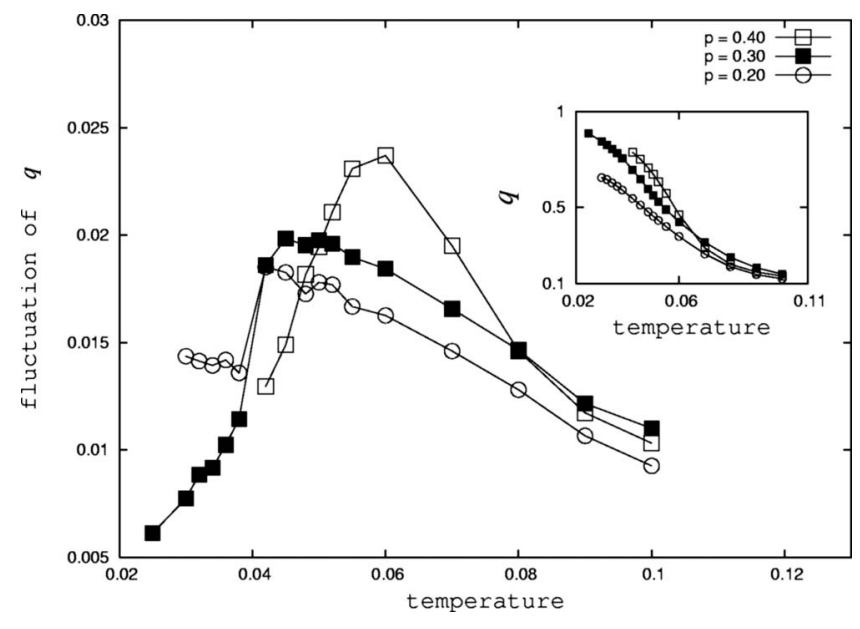

FIG. 5. Variation in the fluctuation of spin-spin correlation $q$ with temperature $T$ for $p=0.40,0.30$, and 0.20 for $N=100$ for classical disordered system. Inset shows the plot of $q$ against temperature $T$.

0.5 , fluctuations $\widetilde{J}$ increase and so is the transition temperature. We have given a plot of the $T_{c} / \tilde{J}$ as a function of $\bar{J} / \widetilde{J}$ (Fig. 6).

\section{LRTIAF with disorder: Quantum model at finite temperature}

\section{Analytical studies}

The approximate saddle-point equations have already been presented in Eqs. (5)-(7). For detail calculations, see Appendix A. The variations in $m^{x}, q$, and $\widetilde{q}$ are shown in Fig. 7. The phase boundary between the spin-glass and paramagnetic phases is given by setting $m^{z}=0$ and $q \simeq 0$ and we get

$$
\Gamma=\widetilde{J} \tanh \left(\frac{\Gamma}{T}\right) .
$$

Obviously, the boundary at $T=0$ gives $\Gamma_{S G}=\widetilde{J}$. On the other hand, when we consider the case of $\Gamma \simeq 0$, we have $T_{S G}=\widetilde{J}$

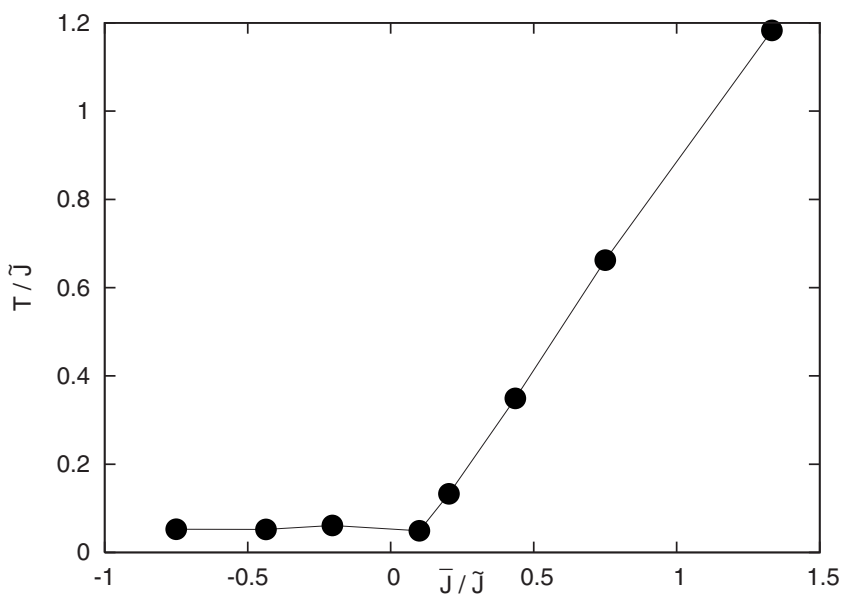

FIG. 6. Phase diagram of classical LRIAF with disorder obtained from Monte Carlo simulation. This phase diagram is similar to that obtained from static and replica-symmetric approximations. 


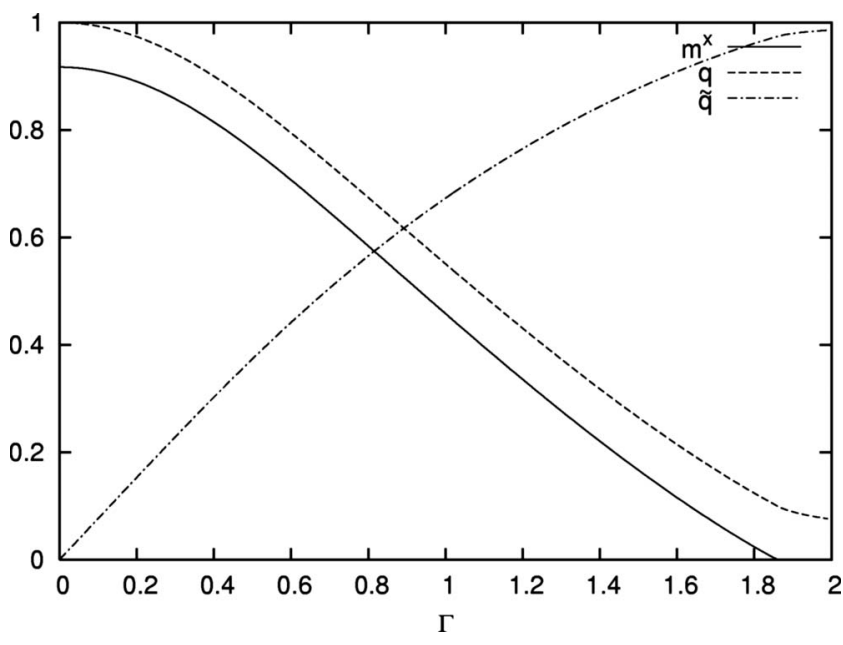

FIG. 7. Result of numerical calculations for the saddle-point equations for $m_{x}, q$, and $\widetilde{q}$ as a function of $\Gamma$ for $T \neq 0$.

(consistent with the classical result). These facts imply that there is neither an antiferromagnetic nor a spin-glass phase when we consider the pure case $\widetilde{J}=0$ because the critical point leads to $T_{S G}=\Gamma_{S G}=0$. Therefore, we conclude that the antiferromagnetic phase can exist if and only if $T=\Gamma=0$ (Fig. 8).

\section{Quantum Monte Carlo studies}

Now to study the quantum system, we again apply the finite-temperature quantum Monte Carlo method as applied to study the pure LRIAF model in Sec. II A Like the classical system, here also we incorporated a disorder with binary distribution $( \pm J)$ with a probability $p$. As mentioned earlier, to study by quantum Monte Carlo, we map the Hamiltonian (1) to a $(\infty+1)$-dimensional classical Hamiltonian using the Suzuki-Trotter formula. The effective Hamiltonian can be written as (for $h=0$ )

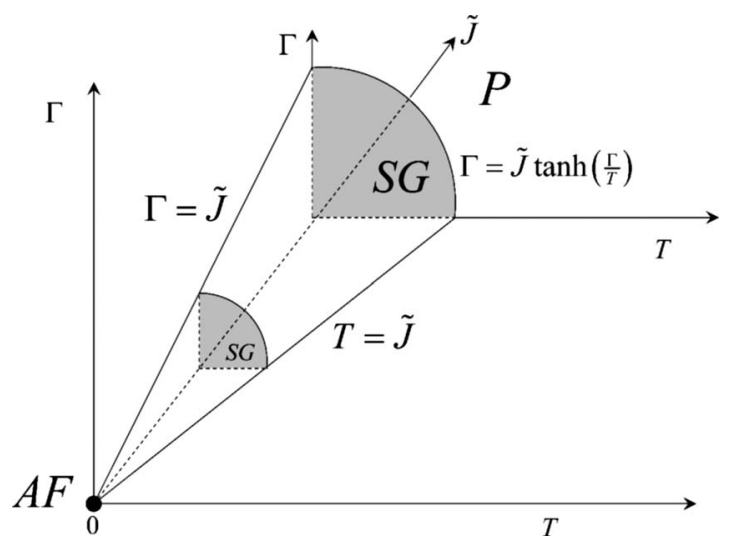

FIG. 8. Phase diagram for the quantum system. The antiferromagnetic order exists if and only if we set $T=\Gamma=0$ and $\widetilde{J}=0$. As the $\widetilde{J}$ decreases, the spin-glass phase gradually shrinks to zero and eventually ends up at an antiferromagnetic phase at its vertex (for $\Gamma=0=T=\widetilde{J})$ as discussed in Sec. II C 1 .

$$
\mathcal{H}=-\frac{1}{N M} \sum_{i, j(>i)=1}^{N} \sum_{k=1}^{M} J_{i j} \sigma_{i, k} \sigma_{j, k}+B \sum_{i=1}^{N} \sum_{k=1}^{M} \sigma_{i, k} \sigma_{i, k+1},
$$

where

$$
B=(1 / 2) \ln [\operatorname{coth}(\Gamma / M T)],
$$

with $M, k$, and $B$ representing the number of Trotter replicas, $k$ th row in Trotter direction, and nearest-neighbor interaction strength along the Trotter direction, respectively. The distribution $\widetilde{P}\left(J_{i j}\right)$ is given by Eq. (20). To identify the glass phase, a replica of the original system to be studied has been taken and the two systems have been evolved simultaneously by Monte Carlo technique.

The quantity measured $(q)$ is the average absolute value of the spin-spin correlations of the two replica systems at different times for a certain temperature and $\Gamma$, where $\alpha$ and $\beta$ denote the original one and the replica

$$
q=\left\langle\left|\frac{1}{N M} \sum_{i=1}^{N M} s_{i}^{\alpha} s_{i}^{\beta}\right|\right\rangle \text {. }
$$

Initially, we kept the temperature fixed at a certain value (typically $T=0.1$ ) and measured the average steady-state values of this parameter for various values of $\Gamma$. The average is over different configurations. Here also, the glass and paramagnetic phases can be distinguished by studying the steadystate values and fluctuation of $q$. For a particular value of $p$, up to a certain $\Gamma$, the value of $q$ remains high, indicating the spin-glass phase. The fluctuations in $q$ remain small. This implies that the flipping of spins is very low (freezing of spins). As the value of $\Gamma$ is raised, the steady-state value of $q$ decreases gradually [inset of Fig. 9(a)], but the fluctuation increases [Fig. 9(a)]. At a certain value of $\Gamma$ (say, $\Gamma_{c}$ ) (glassparamagnetic phase-transition point), this fluctuation reaches maximum and above this $\Gamma_{c}$, the value of $q$ goes to zero. With further increase of $\Gamma$, the fluctuation decreases, indicating a second-order glass-paramagnetic phase transition. The observed values of $\Gamma_{c}$ are $\simeq 0.2,0.35$, and 0.58 for $p=0.2$, 0.3 , and 0.4 , respectively. A similar study for $T=0.2$ is presented in Fig. 9(b). The values of $\Gamma_{c}$ are $\simeq 0.10,0.20$, and 0.40 for $p=0.2,0.3$, and 0.38 , respectively.

It has been noticed that as we increase the value of $p$ from 0 to 0.5 , the transition field increases. It is expected from our previous explanation, as $p$ approaches 0.5 , fluctuations $\widetilde{J}$ increase and so is the transition field $\Gamma_{c}$. We have given a plot of the $\Gamma_{c}$ as a function of $\widetilde{J}$ (Fig. 10). Though these results do not satisfy the predictions of static and replica-symmetric approximations quantitatively, but qualitatively, they reflect all the features

\section{LRTIAF with disorder: Quantum model at zero temperature}

We study this case only analytically. As well known, in the mean-field description of the pure quantum transverse Ising systems, the total magnetization is conserved as $\left(m^{z}\right)^{2}$ $+\left(m^{x}\right)^{2}=1$. However, if some disorders are taken into ac- 

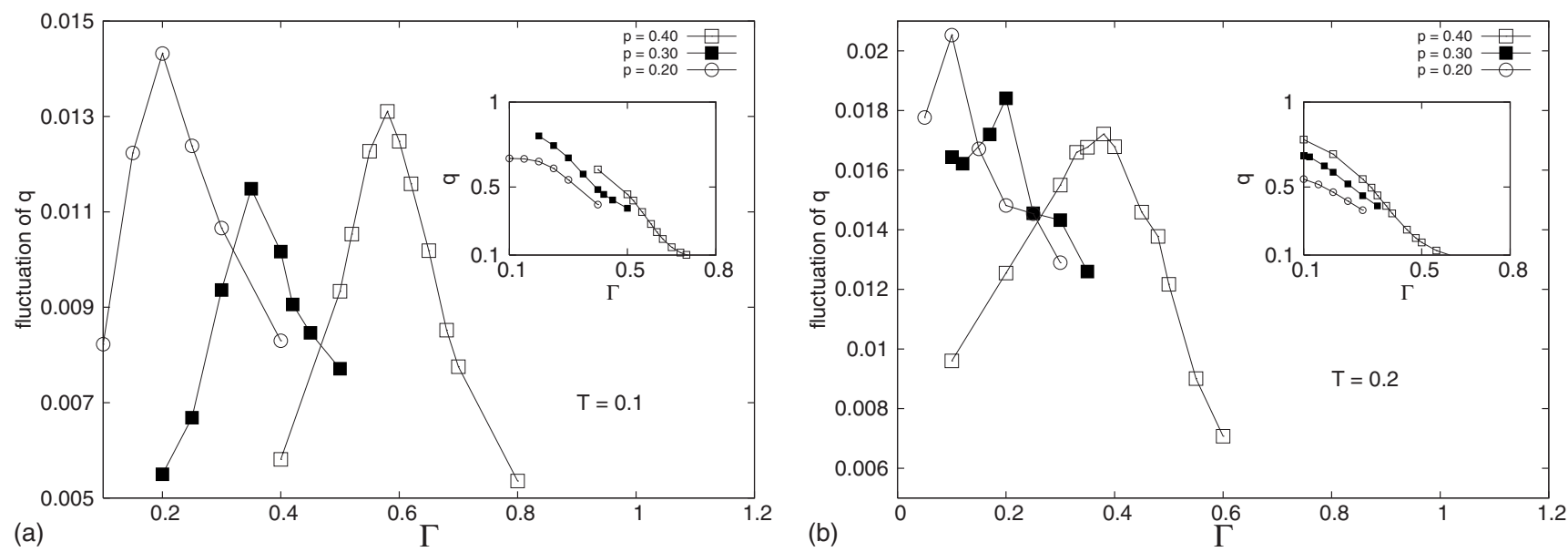

FIG. 9. (a) Variation in the fluctuation of spin-spin correlation $q$ with $\Gamma$ at $T=0.1$ for $p=0.40,0.30$, and 0.20 for $N=100(M=5)$. Inset shows the plot of $q$ against transverse field $\Gamma$. (b) Variation in the fluctuation of spin-spin correlation $q$ with $\Gamma$ at $T=0.2$ for $p=0.40,0.30$, and 0.20 for $N=100$. Inset shows the plot of $q$ against transverse field $\Gamma$.

count, it is nontrivial problem to answer the question; if the magnetization conservation still holds or not. As we discussed before, for antiferromagnets, $m_{z}$ is always zero and the magnetization conservation reads $\left(m^{x}\right)^{2}=1$. In following, we derive the condition on which the magnetization conservation holds.

For finite transverse field but zero temperature, i.e., $\beta$ $=\infty$, Eqs. (8) and (9) reduce to

$$
\begin{gathered}
m^{x}=\int_{-\infty}^{\infty} D y \frac{\Gamma}{\sqrt{(\tilde{J} \sqrt{q} y)^{2}+\Gamma^{2}}}, \\
q=\int_{-\infty}^{\infty} D y\left\{\frac{\tilde{J} \sqrt{q} y}{\sqrt{(\tilde{J} \sqrt{q} y)^{2}+\Gamma^{2}}}\right\}^{2},
\end{gathered}
$$

which are obtained within the replica-symmetric theory in this zero-temperature limit (see Appendix A). The variations

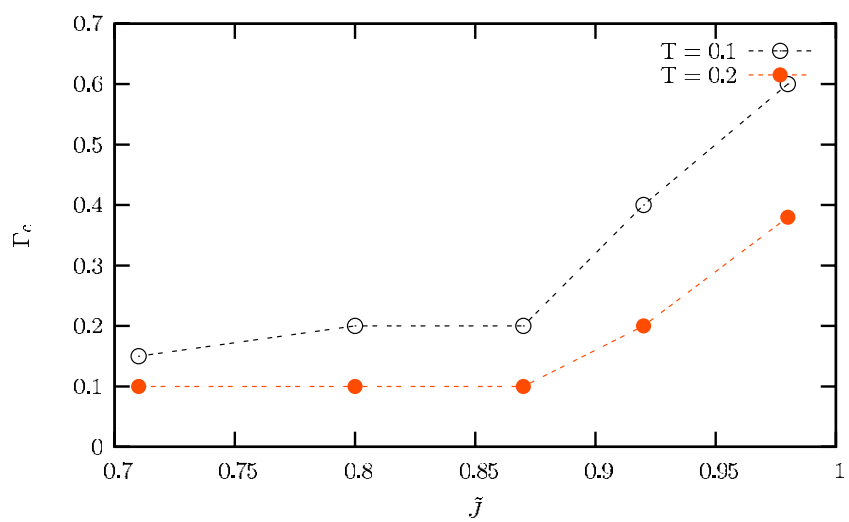

FIG. 10. (Color online) Variation in $\Gamma_{c}$ with $\widetilde{J}$ for the quantum LRIAF with disorder obtained from Monte Carlo simulation. This plot is qualitatively similar to that obtained from static and replicasymmetric approximations. of $m^{x}$ and $q$ are shown in Fig. 11. It can also be seen that, for no disorder, i.e., $\widetilde{J}=0$, we obtain $m^{x}=1$ and $q=0$. For finite disorder $\widetilde{J} \neq 0$, we expand Eq. (27) with respect to $\widetilde{J} \sqrt{q} y \ll 1$. Then, we have $1 \simeq(\widetilde{J} / \Gamma)^{2}-3 q(\widetilde{J} / \Gamma)^{4}$, namely,

$$
q=\frac{\left(\frac{\tilde{J}}{\Gamma}\right)^{2}-1}{3\left(\frac{\tilde{J}}{\Gamma}\right)^{4}}
$$

where we used $\int_{-\infty}^{\infty} D y y^{2}=1$ and $\int_{-\infty}^{\infty} D y y^{4}=3$. Equation (28) implies $q \neq 0$ for $\Gamma<\widetilde{J}$. Hence,

$$
m^{x}=1-\frac{1}{6 \widetilde{J}}\left(\frac{(\tilde{J} / \Gamma)^{2}-1}{\widetilde{J} / \Gamma}\right),
$$

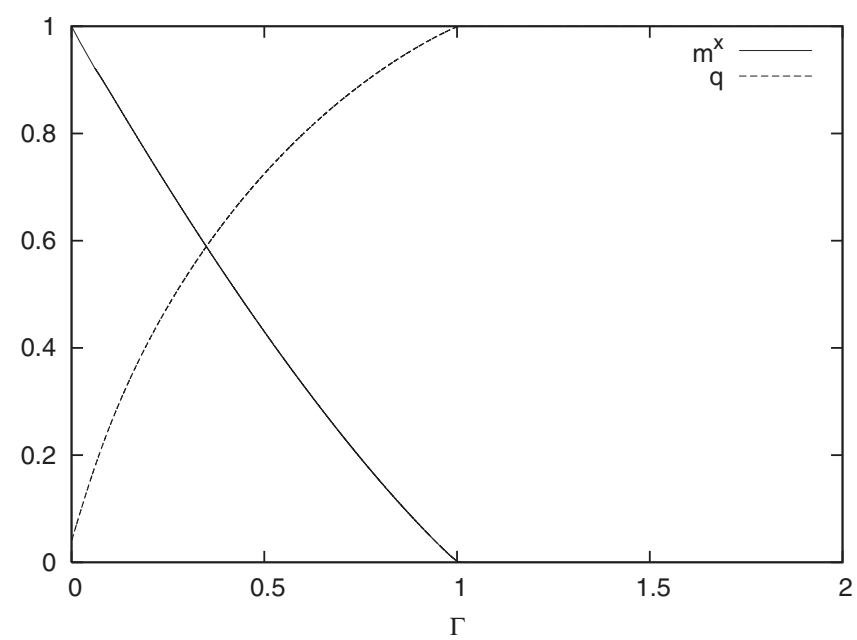

FIG. 11. Result of numerical calculations for the saddle-point equations for $m_{x}$ and $q$ as a function of $\Gamma$ for $T=0$. 


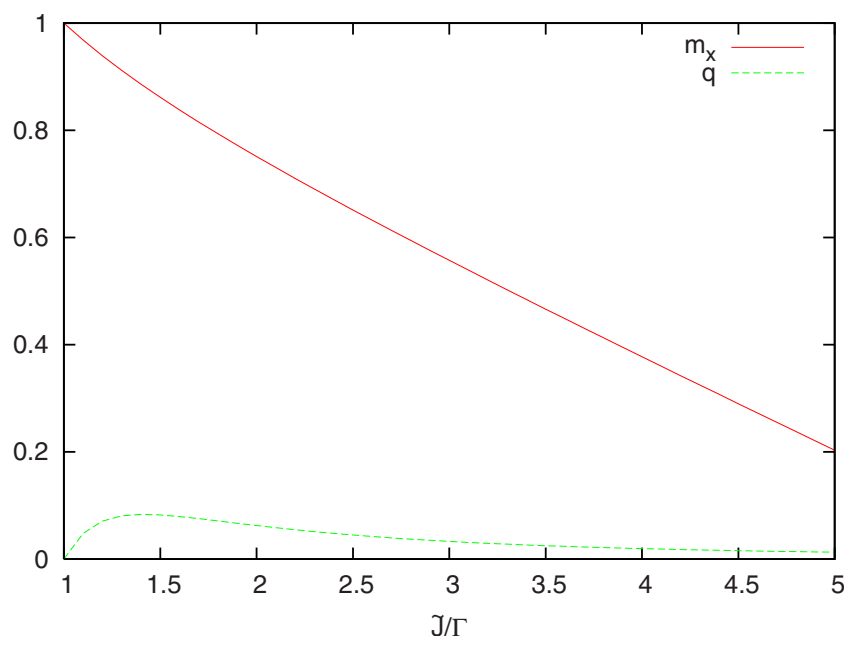

FIG. 12. (Color online) Variation in $m^{x}$ and $q$ with $\widetilde{J} / \Gamma$.

$$
q=\frac{(\widetilde{J} / \Gamma)^{2}-1}{3(\widetilde{J} / \Gamma)^{4}} .
$$

From this result, we find that magnetization conservation is broken for $\Gamma<\widetilde{J}$. This fact means that each spin starts frozen locally and the degree of freedom of spins is remarkably reduced. Therefore, $m^{x}$ might be a good indicator to detect the spin-glass phase for the system in which the both order (antiferromagnet) and disorder (spin-glass) phases possess the same spatial symmetry with $m^{z}=0$ (Fig. 12).

\section{DISCUSSION}

We considered here first a long-range Ising antiferromagnet with disorder incorporated in it and put it in a transverse field. Although long-range interaction is unrealistic and also difficult for numerical studies, it is convenient for analytical approaches such as mean-field calculations which are exact in certain limits. So here we have studied this kind of longrange interaction. We have obtained the finite-temperature free-energy expression (Appendix A) for this model and studied analytically the magnetization, spin-glass order, and correlation (Trotter replica). For the pure case (i.e., no disorder), the antiferromagnetic order is seen to get immediately broken as soon as the thermal or quantum fluctuations are added (see Sec. II A). However, when we add the disorder as in SK Hamiltonian on that of LRTIAF as perturbation, we find that an infinitesimal spin-glass disorder is enough to induce a stable-glass order in this LRTIAF antiferromagnet (Sec. II B). This glass order eventually gets destroyed as the thermal or quantum fluctuations increased beyond their threshold values and the transition to paramagnetic phase occurs (Secs. II B and II C). As shown in the phase diagram in Fig. 4, the antiferromagnetic phase of the LRTIAF (occurring only at $\widetilde{J}=0=\Gamma=T$ ) can get frozen into spin-glass phase if a little SK-type disorder is added $(\widetilde{J} \neq 0)$; the only missing element in the LRTIAF (which is fully frustrated but lacks disorder) to induce stable order (freezing of random-spin orientations) in it. These results have been confirmed by Monte
Carlo simulations. We have not addressed the question of possible replica symmetry breaking in our study. The degeneracy factor $e^{0.693 N}$ of the ground state of the LRIAF is much larger than that $e^{0.199 N}$ for the SK model. Hence, (because of the presence of full frustration), the LRIAF possesses a surrogate incubation property of stable spin-glass phase in it when induced by addition of a small disorder.

\section{ACKNOWLEDGMENTS}

We are grateful to I. Bose, A. Das, S. Dasgupta, D. Sen, P. Sen, and K. Sengupta for useful discussions and comments. One of the authors (J.I.) was financially supported by Grantin-Aid Scientific Research on Priority Areas "Deepening and Expansion of Statistical Mechanical Informatics (DEXSMI)" of the MEXT Grant No. 18079001 and INSA (Indian National Science Academy)-JSPS (Japan Society of Promotion of Science) Bilateral Exchange Program. He also thanks Saha Institute of Nuclear Physics for their warm hospitality during his stay in India. A.K.C. acknowledges Hokkaido University for their hospitality during his stay in Japan.

\section{APPENDIX A: DERIVATION OF FREE ENERGY}

In this appendix, we show the derivation of the free energy per spin for the system to be described by the Hamiltonian

$$
H=-\sum_{i j} J_{i j} \sigma_{i}^{z} \sigma_{j}^{z}-\Gamma \sum_{i} \sigma_{i}^{x} .
$$

Carrying out the Suzuki-Trotter decomposition, we have the replicated partition function

$$
\begin{gathered}
Z_{M}^{n}=\operatorname{tr}_{\{\sigma\}} \exp \left[\frac{\beta}{M} \sum_{i j} \sum_{k} \sum_{\alpha} J_{i j} \sigma_{i}^{\alpha}(k) \sigma_{j}^{\alpha}(k)\right. \\
\left.+B \sum_{i} \sum_{k} \sum_{\alpha} \sigma_{i}^{\alpha}(k) \sigma_{i}^{\alpha}(k+1)\right] \\
B=\frac{1}{2} \ln \operatorname{coth}\left(\frac{\beta \Gamma}{M}\right)
\end{gathered}
$$

where $\alpha$ and $k$ denote the replica and Trotter indices. $M$ is the number of the Trotter slices and $\beta$ is the inverse temperature. The disorder $J_{i j}$ obeys

$$
P\left(J_{i j}\right)=\frac{1}{\sqrt{2 \pi J^{2}}} \exp \left[-\frac{\left(J_{i j}-j_{0}\right)^{2}}{2 J}\right] .
$$

In other words, $J_{i j}$ follows

$$
J_{i j}=j_{0}+J x, \quad P(x)=\frac{1}{\sqrt{2 \pi}} \exp \left(-x^{2} / 2\right) .
$$

We should notice that $j_{0}>0, J=0$ is pure ferromagnetic transverse Ising model, whereas $j_{0}<0, J=0$ corresponds to pure antiferromagnetic transverse Ising model. Then, by using $\int_{-\infty}^{\infty} D x e^{a x}=\exp \left(a^{2} / 2\right), D x \equiv d x \exp \left(-x^{2} / 2\right) / \sqrt{2 \pi}$, we have the average of the replicated partition function as 


$$
\begin{aligned}
\left\langle\left\langle Z_{M}^{n}\right\rangle\right\rangle & =\operatorname{tr}_{\{\sigma\}} \exp \left[\frac{\beta j_{0}}{M} \sum_{k} \sum_{\alpha} \sum_{i j} \sigma_{i}^{\alpha}(k) \sigma_{j}^{\alpha}(k)+B \sum_{k} \sum_{\alpha} \sum_{i} \sigma_{i}^{\alpha}(k) \sigma_{i}^{\alpha}(k+1)\right] \exp \left[\frac{\beta^{2} \widetilde{J}^{2}}{2 M^{2}} \sum_{i j}\left(\sum_{k} \sum_{\alpha} \sigma_{i}^{\alpha}(k) \sigma_{j}^{\alpha}(k)\right)^{2}\right] \\
& =\operatorname{tr}_{\{\sigma\}} \exp \left[\frac{\beta j_{0}}{M} \sum_{k} \sum_{\alpha} \sum_{i j} \sigma_{i}^{\alpha}(k) \sigma_{j}^{\alpha}(k)+B \sum_{k} \sum_{\alpha} \sum_{i} \sigma_{i}^{\alpha}(k) \sigma_{i}^{\alpha}(k+1)\right] \exp \left[\frac{\beta^{2} \widetilde{J}^{2}}{2 M^{2}} \sum_{k k^{\prime}} \sum_{\alpha \beta} \sum_{i j} \sigma_{i}^{\alpha}(k) \sigma_{j}^{\alpha}(k) \sigma_{i}^{\beta}\left(k^{\prime}\right) \sigma_{j}^{\beta}\left(k^{\prime}\right)\right],
\end{aligned}
$$

where the bracket was defined as $\langle\langle\cdots\rangle\rangle=\int \Pi_{i j} d J_{i j} P\left(J_{i j}\right)(\cdots)$. To take a proper thermodynamic limit, we use the scaling

$$
j_{0}=\frac{J_{0}}{N}, \quad J=\frac{\widetilde{J}}{\sqrt{N}} .
$$

For this rescaling of the parameters, the averaged replicated partition function $\left\langle\left\langle Z_{M}^{n}\right\rangle\right\rangle$ reads

$$
\begin{aligned}
\left\langle\left\langle Z_{M}^{n}\right\rangle\right\rangle= & \operatorname{tr}_{\{\sigma\}} \int_{-\infty}^{\infty} \prod_{k} \prod_{\alpha} \frac{d m_{\alpha}(k)}{\sqrt{2 \pi M / \beta J_{0} N}} \int_{-\infty}^{\infty} \prod_{k k^{\prime}} \prod_{\alpha \beta} \frac{d q_{\alpha \beta}\left(k, k^{\prime}\right)}{\sqrt{2 \pi M / \beta \widetilde{J} \sqrt{N}}} \int_{-\infty}^{\infty} \prod_{k k^{\prime}} \prod_{\alpha} \frac{d \widetilde{q}_{\alpha \alpha}\left(k, k^{\prime}\right)}{\sqrt{2 \pi M / \beta \widetilde{J} \sqrt{N}}} \\
& \times \exp \left[-\frac{\beta J_{0} N}{2 M} \sum_{k} \sum_{\alpha} m_{\alpha}(k)^{2}-\frac{(\beta \widetilde{J})^{2} N}{2 M^{2}} \sum_{k k^{\prime}} \sum_{\alpha \beta} q_{\alpha \beta}\left(k, k^{\prime}\right)^{2}-\frac{(\beta \widetilde{J})^{2} N}{2 M^{2}} \sum_{k k^{\prime}} \sum_{\alpha} \widetilde{q}_{\alpha \alpha}\left(k, k^{\prime}\right)^{2}\right] \exp \left[\frac{\beta J_{0}}{M} \sum_{k} \sum_{\alpha} m_{\alpha}(k) \sum_{i} \sigma_{i}^{\alpha}(k)\right. \\
& \left.+\left(\frac{\beta \widetilde{J}}{M}\right)^{2} \sum_{k k^{\prime}} \sum_{\alpha \beta} q_{\alpha \beta}\left(k, k^{\prime}\right) \sum_{i} \sigma_{i}^{\alpha}(k) \sigma_{i}^{\beta}\left(k^{\prime}\right)+\left(\frac{\beta \widetilde{J}}{M}\right)^{2} \sum_{k k^{\prime}} \sum_{\alpha} \widetilde{q}_{\alpha \alpha}\left(k, k^{\prime}\right) \sum_{i} \sigma_{i}^{\alpha}(k) \sigma_{i}^{\alpha}\left(k^{\prime}\right)+B \sum_{k} \sum_{\alpha} \sum_{i} \sigma_{i}^{\alpha}(k) \sigma_{i}^{\alpha}(k+1)\right] .
\end{aligned}
$$

We next assume the replica symmetry and static approximations such as

$$
\begin{gathered}
m_{\alpha}(k)=\left\langle\sigma^{\alpha}(k)\right\rangle=\frac{1}{N} \sum_{i} \sigma_{i}^{\alpha}(k)=m, \\
q_{\alpha \beta}\left(k, k^{\prime}\right)=\left\langle\sigma^{\alpha}(k) \sigma^{\beta}\left(k^{\prime}\right)\right\rangle=\frac{1}{N} \sum_{i} \sigma_{i}^{\alpha}(k) \sigma_{i}^{\beta}\left(k^{\prime}\right)=q, \\
\widetilde{q}_{\alpha \alpha}\left(k, k^{\prime}\right)=\left\langle\sigma^{\alpha}(k) \sigma^{\alpha}\left(k^{\prime}\right)\right\rangle=\frac{1}{N} \sum_{i} \sigma_{i}^{\alpha}(k) \sigma_{i}^{\alpha}\left(k^{\prime}\right)=\widetilde{q} .
\end{gathered}
$$

Then, we should notice the relation

$$
\begin{aligned}
\left(\frac{\beta \widetilde{J}}{M}\right)^{2} q \sum_{k k^{\prime}} \sum_{\alpha \beta} \sum_{i} \sigma_{i}^{\alpha}(k) \sigma_{i}^{\beta}\left(k^{\prime}\right) \\
=\left(\frac{\beta \widetilde{J}}{M}\right)^{2} q \sum_{i}\left(\sum_{k} \sum_{\alpha} \sigma_{i}^{\alpha}(k)\right)^{2} \\
-\left(\frac{\beta \widetilde{J}}{M}\right)^{2} q \sum_{i} \sum_{\alpha}\left(\sum_{k} \sigma_{i}^{\alpha}(k)\right)^{2},
\end{aligned}
$$

$$
\left(\frac{\beta \tilde{J}}{M}\right)^{2} \tilde{q} \sum_{k k^{\prime}} \sum_{\alpha} \sum_{i} \sigma_{i}^{\alpha}(k) \sigma_{i}^{\alpha}\left(k^{\prime}\right)=\left(\frac{\beta \tilde{J}}{M}\right)^{2} \tilde{q} \sum_{i} \sum_{\alpha}\left(\sum_{k} \sigma_{i}^{\alpha}(k)\right)^{2} .
$$

To take into account the above relations, we obtain in the limit of $N \rightarrow \infty$ as

$$
\begin{aligned}
\left\langle\left\langle Z_{M}^{n}\right\rangle\right\rangle= & \int_{-\infty}^{\infty} \prod_{k} \prod_{\alpha} \frac{d m_{\alpha}(k)}{\sqrt{2 \pi M / \beta J_{0} N}} \int_{-\infty}^{\infty} \prod_{k k^{\prime}} \prod_{\alpha \beta} \frac{d q_{\alpha \beta}\left(k, k^{\prime}\right)}{\sqrt{2 \pi M / \beta \widetilde{J} \sqrt{N}}} \int_{-\infty}^{\infty} \prod_{k k^{\prime}} \prod_{\alpha} \frac{d \widetilde{q}_{\alpha \alpha}\left(k, k^{\prime}\right)}{\sqrt{2 \pi M / \beta \widetilde{J} \sqrt{N}}} \\
& \times \exp \left[n N\left(-\frac{\beta J_{0}}{2} m^{2}+\frac{(\beta \widetilde{J})^{2}}{4} q^{2}-\frac{(\beta \widetilde{J})^{2}}{4} \widetilde{q}^{2}+\int_{-\infty}^{\infty} D y \ln \int_{-\infty}^{\infty} D u 2 \cosh \beta \sqrt{\left(J_{0} m+\widetilde{J} \sqrt{q} y+\widetilde{J} \sqrt{\widetilde{q}-q} u\right)^{2}+\Gamma^{2}}\right)\right] \\
\simeq & \exp [n N f] .
\end{aligned}
$$


Therefore, the following $f$ is regarded as free energy per spin by the definition of replica theory:

$$
\begin{aligned}
f= & -\frac{\beta J_{0}}{2} m^{2}+\frac{(\beta \widetilde{J})^{2}}{4} q^{2}-\frac{(\beta \widetilde{J})^{2}}{4} \tilde{q}^{2}+\int_{-\infty}^{\infty} D y \ln \int_{-\infty}^{\infty} D u 2 \\
& \times \cosh \beta \sqrt{\left(J_{0} m+\widetilde{J} \sqrt{q} y+\widetilde{J} \sqrt{\tilde{q}-q} u\right)^{2}+\Gamma^{2}}
\end{aligned}
$$

\section{Saddle point equations}

For simplicity, we define

$$
\begin{gathered}
b=J_{0} m+\widetilde{J} \sqrt{q} y+\widetilde{J} \sqrt{\widetilde{q}-q} u, \\
\Theta=\sqrt{b^{2}+\Gamma^{2}} .
\end{gathered}
$$

Then, we have the following simplified free energy:

$f=-\frac{\beta J_{0}}{2} m^{2}+\frac{(\beta \widetilde{J})^{2}}{4}\left(q^{2}-\widetilde{q}^{2}\right)+\int_{-\infty}^{\infty} D y \ln \int_{-\infty}^{\infty} D u 2 \cosh \beta \Theta$.

The saddle-point equations are derived as follows $[15,16]$ :

$$
\begin{gathered}
m=\int_{-\infty}^{\infty} D y\left[\frac{\int_{-\infty}^{\infty} D u\left(\frac{b}{\Theta}\right) \sinh \beta \Theta}{\int_{-\infty}^{\infty} D u \cosh \beta \Theta}\right], \quad \text { (A19) } \\
q=\int_{-\infty}^{\infty} D y\left[\frac{\int_{-\infty}^{\infty} D u\left(\frac{b}{\Theta}\right) \sinh \beta \Theta}{\int_{-\infty}^{\infty} D u \cosh \beta \Theta}\right], \quad(\mathrm{A} 20) \\
\widetilde{q}=\int_{-\infty}^{\infty} D y\left[\frac{\int_{-\infty}^{\infty} D u\left\{\left(\frac{b^{2}}{\Theta^{2}}\right) \cosh \beta \Theta+\frac{\Gamma \beta^{-1}}{\Theta^{3}} \sinh \beta \Theta\right\}}{\int_{-\infty}^{\infty} D u \cosh \beta \Theta}\right], \\
m_{x}=\frac{\partial f}{\partial \Gamma}=\int_{-\infty}^{\infty} D y\left[\frac{\int_{-\infty}^{\infty} D u\left(\frac{\Gamma}{\Theta}\right) \sinh \beta \Theta}{\int_{-\infty}^{\infty} D u \cosh \beta \Theta}\right] . \quad(\mathrm{A} 22)
\end{gathered}
$$

\section{At the ground state}

We first should notice that $\widetilde{q}$ is always larger than $q$. In fact, we can easily show that

$$
\begin{aligned}
& \tilde{q}=\int_{-\infty}^{\infty} D y\left[\frac{\int_{-\infty}^{\infty} D u\left(\frac{b^{2}}{\Theta^{2}}\right) \cosh \beta \Theta}{\int_{-\infty}^{\infty} D u \cosh \beta \Theta}\right] \\
& \geq \int_{-\infty}^{\infty} D y\left[\frac{\int_{-\infty}^{\infty} D u\left(\frac{b^{2}}{\Theta^{2}}\right) \sinh \beta \Theta}{\int_{-\infty}^{\infty} D u \cosh \beta \Theta}\right] \\
& \geq \int_{-\infty}^{\infty} D y\left[\frac{\int_{-\infty}^{\infty} D u\left(\frac{b^{2}}{\Theta^{2}}\right) \sinh \beta \Theta}{\int_{-\infty}^{\infty} D u \cosh \beta \Theta}\right]=q .
\end{aligned}
$$

Then, we consider the limit of $\beta \rightarrow \infty$. If $\widetilde{q}-q=\epsilon \geq 0$ is of order 1 object, the free energy $f$ diverges in the limit of $\beta$ $\rightarrow \infty$ as $(\beta \widetilde{J})^{2}\left(q^{2}-\widetilde{q}^{2}\right) / 4$. Therefore, we conclude that $q=\widetilde{q}$ should be satisfied in the limit of $\beta \rightarrow \infty$ and we obtain the saddle-point equation at the ground state as

$$
m=\int_{-\infty}^{\infty} D y\left(\frac{b}{\Theta}\right)=\int_{-\infty}^{\infty} D y \frac{\left(J_{0} m+\widetilde{J} \sqrt{q} y\right)}{\sqrt{\left(J_{0} m+\widetilde{J} \sqrt{q} y\right)^{2}+\Gamma^{2}}},
$$

$$
\begin{gathered}
q=\widetilde{q}=\int_{-\infty}^{\infty} D y\left(\frac{b}{\Theta}\right)^{2}=\int_{-\infty}^{\infty} D y\left\{\frac{\left(J_{0} m+\widetilde{J} \sqrt{q} y\right)}{\sqrt{\left(J_{0} m+\widetilde{J} \sqrt{q} y\right)^{2}+\Gamma^{2}}}\right\}^{2}, \\
m_{x}=\int_{-\infty}^{\infty} D y\left(\frac{\Gamma}{\Theta}\right)=\int_{-\infty}^{\infty} D y \frac{\Gamma}{\sqrt{\left(J_{0} m+\widetilde{J} \sqrt{q} y\right)^{2}+\Gamma^{2}}} .
\end{gathered}
$$

\section{APPENDIX B: EXACT ANALYSIS AT $T=0$}

First, let us consider the case of pure LRIAF model and rewrite our Hamiltonian $H$ in Eq. (1) for $\widetilde{J}=0$ as

$$
H=\frac{1}{2 N}\left(\sum_{i=1}^{N} \sigma_{i}^{z}\right)^{2}-\frac{1}{N} \sum_{i=1}^{N}\left(\sigma_{i}^{z}\right)^{2}-h \sum_{i=1}^{N} \sigma_{i}^{z}-\Gamma \sum_{i=1}^{N} \sigma_{i}^{x} .
$$

If we now denote the total spin by $\vec{\sigma}_{t o t}$, i.e., $\vec{\sigma}_{t o t}=\frac{1}{N} \sum_{i=1}^{N} \vec{\sigma}_{i}$ (where $N|\vec{\sigma}|=0,1,2, \ldots, N)$, then the Hamiltonian $H$ can be expressed as

$$
\frac{H}{N}=\frac{1}{2}\left(\sigma_{t o t}^{z}\right)^{2}-h \sigma_{t o t}^{z}-\Gamma \sigma_{t o t}^{x}-\frac{1}{N} .
$$

Let us assume the average total spin $\langle\vec{\sigma}\rangle$ to be oriented at an angle $\theta$ with the $z$ direction: $\left\langle\sigma_{t o t}^{z}\right\rangle=m \cos \theta$ and $\left\langle\sigma_{t o t}^{x}\right\rangle$ $=m \sin \theta$. Hence, the average total energy $E_{\text {tot }}=\langle H\rangle$ can be written as 


$$
\frac{E_{t o t}}{N}=\frac{1}{2} m^{2} \cos ^{2} \theta-h m \cos \theta-\Gamma m \sin \theta-\frac{1}{N} .
$$

At the zero temperature and at $\Gamma=0$, for $h=0$, the energy $E_{\text {tot }}$ is minimized when $\theta=0$ and $m=0$ (complete antiferromagnetic order in $z$ direction). As soon as $\Gamma \neq 0(h=0)$, the minimization of $E_{\text {tot }}$ requires $\theta=\pi / 2$ and $m=1$ (the maximum possible value); driving the system to paramagnetic phase. This discontinuous transition at $T=0$ was also seen in [9]. As observed in our Monte Carlo study in the previous section, $\Gamma_{c}(T) \rightarrow 0$ as $T \rightarrow 0$. This is consistent with this exact result $\Gamma_{c}=0$ at $T=0$. For $T=0$ (and $h=0$ ), therefore, the transition from antiferromagnetic $(\theta=0=m)$ to paramagnetic $(\theta=\pi / 2, m=1)$ phase, driven by the transverse field $\Gamma$, occurs at $\Gamma=0$ itself.

One can also calculate the susceptibility $\chi$ at $\Gamma=0=T$. Here, $E_{t o t} / N=\frac{1}{2} m^{2} \cos ^{2} \theta-h m \cos \theta-\frac{1}{N}$ and the minimization of this energy gives $m \cos \theta=h$, giving the (longitudinal) susceptibility $\chi=m \cos \theta / h=1$. This is consistent with the observed behavior of $\chi$ shown in Fig. 2 where the extrapolated value of $\chi$ at $\Gamma=0$ increases with decreasing $T$ and approaches $\chi=1$ as $T \rightarrow 0$.

At finite temperatures $T \neq 0$, for $h=0$, we have to consider also the entropy term and minimize the free energy $\mathcal{F}=E_{\text {tot }}$ - TS rather than $E_{t o t}$, where $S$ denotes the entropy of the state. This entropy term will also take part in fixing the value of $\theta$ and $m$ at which the free energy $\mathcal{F}$ is minimized. As soon as the temperature $T$ becomes nonzero, the extensive entropy of the system for antiferromagnetically ordered state with $m \simeq 0$ (around and close-by excited states with $\theta=0$ ) helps stabilization near $\theta=0$ and $m=0$ rather than near the paramagnetic phase with $\theta=\pi / 2$ and $m=1$, where the entropy drops to zero. While the transverse field tends to align the spins along $x$ direction (inducing $\theta=\pi / 2$ and $m=1$ ), the entropy factor prohibits that and the system adjusts $\theta$ and $m$ values accordingly and they do not take the disordered or paramagnetic state values $(\theta=\pi / 2$ and $m=1)$ for any nonzero value of $\Gamma$ (like at $T=0$ ). For very large values of $\Gamma$, of course, the free energy $\mathcal{F}$ is practically dominated by the transverse field term in $H$ and again $\theta=\pi / 2$ and $m=1$, beyond $\Gamma=\Gamma_{c}(T)>0$ for $T>0$. However, this continuoustransition-like behavior may be argued [17] to correspond to a crossover-type property of the model at finite temperatures [suggesting that the observed finite values of $\Gamma_{c}(T)$ are only effective numerical values]. In fact, for $h=0$, one adds the entropy term $-T \ln D$, where $D$ is the degeneracy for getting total spin $\tau=N\left|\vec{\sigma}_{\text {tot }}\right|[9]$,

$$
D=\frac{N !}{(N / 2+\tau) !(N / 2-\tau) !}-\frac{N !}{(N / 2+\tau+1) !(N / 2-\tau-1) !},
$$

to $E_{\text {tot }}$ in Eq. (A3) to get $\mathcal{F}$ and one can then get, after minimizing the $\mathcal{F}$ with respect to $m$ and $\theta, m=\tanh (\Gamma / 2 T)$, which indicates an analytic variation in $m$ and no phase transition at any finite temperature for $\widetilde{J}=0$ (antiferromagnetic phase occurs only at $\Gamma=T=0$ and $J_{0} / \widetilde{J}=\infty$ as shown in Fig. 4).
[1] R. N. Bhatt, in Spin Glasses and Random Fields, edited by A. P. Young (World Scientific, Singapore, 1998), p. 225.

[2] B. K. Chakrabarti, A. Dutta, and P. Sen, Quantum Ising Phases and Transitions in Transverse Ising Models (Springer-Verlag, Heidelberg, 1996).

[3] N. D. Mackenzie and A. P. Young, J. Phys. C 16, 5321 (1983).

[4] Y. Ma and Z. Li, Phys. Lett. A 145, 19 (1990).

[5] A. Canning, J. Phys. A 25, 4723 (1992).

[6] D.-H. Kim and J.-J. Kim, Phys. Rev. B 66, 054432 (2002).

[7] B. K. Chakrabarti and J.-I. Inoue, Indian J. Phys. 80, 609 (2006).

[8] B. K. Chakrabarti, A. Das, and J.-I. Inoue, Eur. Phys. J. B 51, 321 (2006).

[9] J. Vidal, R. Mosseri, and J. Dukelsky, Phys. Rev. A 69, 054101 (2004).
[10] M. Suzuki, Prog. Theor. Phys. 56, 2454 (1976); B. K. Chakrabarti and A. Das, in Quantum Annealing and Related Optimization Methods, edited by A. Das and B. K. Chakrabarti (Springer, Heidelberg, 2005).

[11] A. K. Chandra, J.-I. Inoue, and B. K. Chakrabarti, J. Phys.: Conf. Ser. 143, 012013 (2009).

[12] A. Ganguli and S. Dasgupta, Phys. Rev. E 80, 031115 (2009).

[13] K. Binder and A. P. Young, Rev. Mod. Phys. 58, 801 (1986).

[14] A. Das and B. K. Chakrabarti, Rev. Mod. Phys. 80, 1061 (2008).

[15] D. Thirumalai, Q. Li, and T. R. Kirkpatrick, J. Phys. A 22, 3339 (1989).

[16] Y. Y. Goldschmidt and P.-Y. Lai, Phys. Rev. Lett. 64, 2467 (1990).

[17] D. Sen (private communication). 